\title{
A measurement technique for stability as applied in rifle shooting
}

\author{
CLAUDE ALAIN and GUY AVON \\ Département de Kinanthropologie, Université du Québec à Montréal, Montreal, Que Canada
}

A technique is described for the measurement, in the field, of stability and steadiness. Apparatus and method are provided along with suggested scoring procedures.

In many perceptual motor skills. stability during the execution of the task can markedly affect performance. Several existing instruments can be used to evaluate stability for gross and fine motor skills. e.g.. stabilometers. steadiness testers. However. these instruments can only be used in experimental settings that are somewhat different, if not entirely unrelated. to the real-life situations faced by the subject. This limitation can be serious since the type of stability measured in such experimental situations could differ from the one imposed by real-life task demands.

The measuring device presented here was elaborated to meet two criteria: (1) it should provide a means to accurately evaluate micro displacements occurring during the task, and (2) it should enable the experimenter to place the subject in an experimental situation that simulates the real-life conditions in which the task is normally accomplished.

The illustration of this technique will be done in light of one of its possible applications: the measurement of fine stability in rifle shooting. Other applications of the technique will be mentioned in the concluding comments.

\section{METHOD}

\section{The Basis of the Technique and the Experimental Setting}

The technique presented here is based on the registering and the analysis of micro displacements obtained via a photographic procedure. As applied here. the technique was used to register the micro displacements occurring at the end of a rifle barrel during the task of shooting. A transmitting device was attached to the end of the barrel and any frontal displacement of the rifle caused a corresponding displacement of the transmitter. The nature of this transmitting device was such that its displacements could be photographed.

The experimental environment in which the registrations of the micro displacements were taken is represented in Figure 1. The subject was asked to position himself in his usual aiming stance and to pretend he was shooting at a target located $8 \mathrm{~m}$ away. He was told to take the time needed to concentrate and to initiate the readiness process he normally used prior to pulling the trigger. The subject verbally signaled the experimenter to indicate optimal

The authors wish to express their appreciation to Mr. Guy Delforge who solved many of the technical problems encountered in the conception and realization of the apparatus.

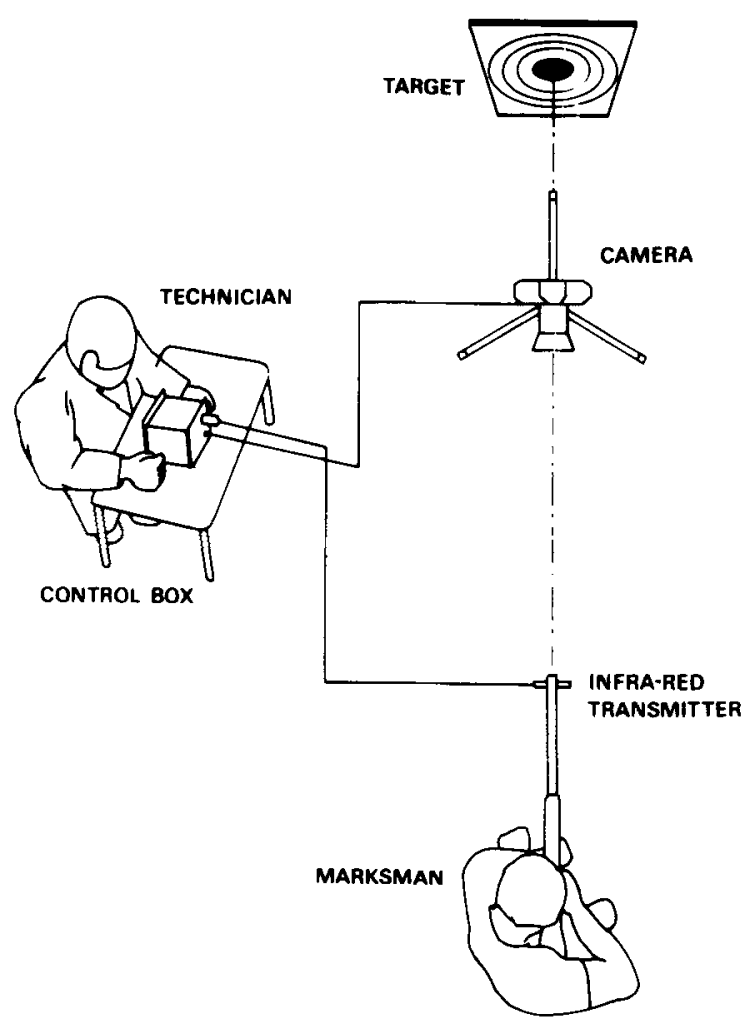

Figure 1. Overhead view of the experimental setting.

stability conditions. At this point, registration of the micro displacements was initiated for a period of either 1. 3. or $5 \mathrm{sec}$. During this period, the micro displacements were photographed by the still picture echnique using a standard camera. The camera was located $j i, i$ below the aiming line and $5 \mathrm{~m}$ away from the transmitting sevice attached to the end of the barrel. This system was operatet at a distance via a control module related to the camera and to the transmitter so that both of these instruments were activated at the same time (Figure 1).

\section{The Apparatus and Its Operation}

Registration by photography can be done by using panchromatic film or an intrared process. The latter was chosen because it made possible the isolatiol. of the signal from its background and yielded a high signal to background contrast in both normal interior and exterior lighting conditions.

The transmitting device attached to the end of the barrel transmits intrared signals. It consists of three infrared diodes 

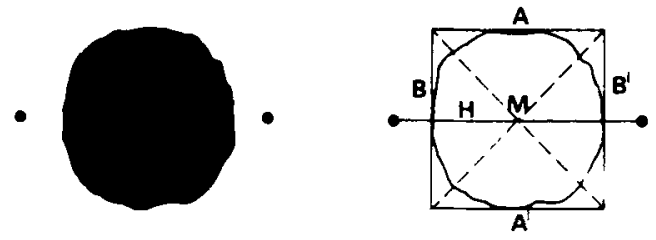

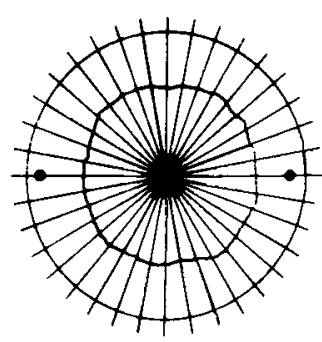

Figure 2. Three-stage process of the registered micro displacements: (a) on the left, registration of the tracing and witness diodes as it appeared on the negative; (b) in center, determination of the middle point on the depicted area; (c) on the right, the 36 distance lengths representing the deviations from the middle point.
(MONSATO. IR-LED ME4) inserted into an aluminum frame. o: $\times 5 \times 5 \mathrm{~mm}$. The device weighs approximately $5 \mathrm{~g}$. Three holes $(.3 \mathrm{~mm}$ diam) were drilled in the anterior face of the frame in order to channel the infrared rays from the diodes. One hole was drilled in the middle of the frame and the two others on each side at $25 \mathrm{~mm}$ from the middle. The diode corresponding to the middle hole. the TRACING DIODE, was activated during the entire length of the registration. i.e.. 1, 3, or $5 \mathrm{sec}$, allowing us to photograph micro displacements occurring at the end of the barrel continually. The other two diodes. the WITNESS DIODES. were activated at the beginning of the registration for $1 / 100$ of a second only. (Because of related measurement problems, the use of the WITNESS DIODES will be elaborated upon in the section dealing with the scoring procedures.)

Three leads of minimal weight connected the three diodes of the transmitting device to a control box which. in turn. was connected to a power supply (Figure 1). The function of the control box was to feed the diodes with a constant current of $100 \mathrm{~mA}$ and to synchronize the activation of both the transmitting device and the camera.

The camera was a Pentax SP II with lens SMCT 83.5 of $135 \mathrm{~mm}$ to which a $20-\mathrm{mm}$ extension tube and a Vivitar $2 \mathrm{X}$ tele-extendet were added; the camera was placed $5 \mathrm{~m}$ away from the transmitting device. A reduction of five times the actual size of the transmitting device was obtained. An infrared tilter (Pentax No. 25) was added to filter extraneous light. This resulted in an ASA of 50 with Kodak high-speed infrared film. The diaphragm was open at $f 16$, and the time shutter turned to "B." The film was developed in a D-19 (Kodak) solution with maximum contrast according to the instructions of the maker.

\section{Measurement of the Micro Displacements}

Each negative. so obtained. one for every trial, was inserted in a frame and used as a slide. To enable measurement of the micro displacements, each slide underwent three processing stages.

First. the slide was magnified approximately 100 times by projecting it onto a white wall. Figure 2 (the far left portion) illustrates one such projection. The large black area in the middle was produced by the registered micro displacements of the TRACING DIODE. The dots on each side of the black area were produced by activation of the WITNESS DIODES. These two dots were used as reference points in order to standardize the size of the magnified pictures. The distance separating the projector from the wall was adjusted in such a way that. for every slide projected, the reference points were $100 \mathrm{~cm}$ apart as measured on the wall. The necessity of such a standardization was to evade a confounding factor inherent in the experimental task (i.e., the distance separating the end of the barrel and the camera could vary from subject to subject because of such factors as arm length, and from trial to trial because of slight movements from rear to front). Registering these reference points enabled the experimenter to establish a standard from which the size of the magnified micro displacements could be compared from subject to subject, and from trial to trial.

The second processing stage consisted of rubbing down, on a transparent sheet of paper, the edge of the projected area which represented the micro displacements during that particular trial. This was done to facilitate the operations which constituted the subsequent and final processing stage.
These operations were the determination of the middle point of the rubbed down area and the measurement of the size of the deviations from this middle point. The middle point was established as follows (Figure 2, center): (1) The horizontal axis was determined by joining the reference points with a straight line, Line $H$. The upper and lower points were located on the depicted area and a line, parallel to the horizontal axis, was drawn from each. Lines $A$ and $A^{\prime}$, respectively. (2) The extreme left and right points were located on the depicted area and. from each, a line was drawn perpendicular to the horizontal axis, Lines $B$ and $B^{\prime}$. respectively. As shown in the figure, Lines $A . A^{\prime}, B$. and $B^{\prime}$ formed a rectangle. (3) The middle point. Point $M$, was given by the intersection of the diagonals of the rectangle.

Taking Point $M$ as its central point, a circle was traced whose radius was greater than the largest deviation from Point $M$ that could be found on the depicted area (Figure 2, on the right). Thirty-six radii, one every $10 \mathrm{deg}$. were traced, each intersecting with the area delimited by the displacements. This resulted in 36 measurable distances representing the magnitude of the deviations from Point $M$.

It is from these deviations and from this last figure that stability scores were derived.

\section{STABILITY SCORES}

Fine stability was operationally defined by the area covered by the micro displacements of the end of the barrel during the registration period; the greater the surface covered, the lower the stability, and vice versa. Once the manipulations described above had been completed and the 36 radii lengths had been obtained, the surface of the area was easily computed by applying the formula $\pi \bar{R}^{2}$, where $\bar{R}$ is the average of the 36 radii lengths $(\Sigma \mathrm{R} / 36)$.

Additional information about the stability performance would be gained if the shape and orientation of the surface covered by the micro displacements could be assessed.

Relative to the shape of the surface, the standard deviation of the radii lengths would give a numerical value reflecting how close the figure is from a perfect circle. Equal radii lengths will give a perfect circle and will also lead to a small standard deviation. Alternately, greater differences between radii lengths will correspond to larger irregularities in the figure and will also lead to a larger standard deviation.

To specify the orientation of the surface, that is. whether the area covered by the micro displacements is spread horizontally, vertically, or otherwise, the Cartesian system can be used. In so doing, the middle point of the depicted area (Figure 2, center) is taken as the origin of the ordinate and the abscissa. The average value of the radii lengths falling in each 
separate quadrant is computed. This gives four average values. These average values are then summed two by two. yielding six summations, one for every combination of quadrants. The combination of the quadrants corresponding to the highest summation represents the predominant orientation of the micro displacements, i.e.. whether the area is spread horizontally, vertically, or obliquely. This merely indicates a trend but does not in any way reflect its size. To find out the relative importance of that trend, the highest summation value has to be compared with the smallest summation obtained. The ratio existing between these two describes the importance of the deviation in relation to its opposed axis.

\section{CONCLUDING COMMENTS}

The measuring technique presented herewith was illustrated in the context of rifle shooting. It can be used in similar fieid testing situations such as pistol shooting and archery. The transmitting device can be affixed to some part of the body, as opposed to some external object. For that reason, the technique can also be used to evaluate stability performance in situations where steadiness of the body or of some part(s) of the body has to be achieved. This is the case, for instance, in some of the gymnastics skills.

We foresee research projects concerned with factors susceptible of affecting stability performance of specific skills because the technique enables stability measurement in situ. The influence of hypnosis, or drugs, or augmented feedback on the stability performance in field testing situations are examples of such projects.

(Received for publication January 8, 1976.) 